\title{
Asymptomatic Infections: The Hidden Epidemic
}

\section{Israel Potasman}

Maccabi HMO, and the Rappaport Faculty of Medicine, Technion, Haifa, Israel

\section{Abstract}

Asymptomatic infectious diseases (AI) are frequent, occasionally even more common than overt febrile presentations. Yet, the literature covering this topic is scarce. Acknowledging the role of AI is crucial both to public health practitioners, because AI silently perpetuate epidemics, such as influenza or malaria, and also crucial to newborns' health and their long-term sequelae.

The recent developments in metagenomics and rapid diagnostics open new horizons for the study of AI. The purpose of this review is to highlight key elements in the epidemiology, mechanisms and implications of well-studied infectious agents: Plasmodium, T. gondii, C. burnetii, Cytomegalovirus, Rhinovirus and Zika virus.

\section{Introduction}

For centuries, physicians investigating infectious diseases have focused on overt infections, namely those presenting with fever, chills, aches, rash, cough, and diarrhea. However, as the tip of the iceberg, every symptomatic case may represent several asymptomatic cases. Asymptomatic infections span the entire microbiome: viruses [1], bacteria [2], parasites [3], and fungi [4]. The case of Mary Mallon, better known as Typhoid Mary, the first person in the United States identified as an asymptomatic carrier of Salmonella, is a formidable example of the importance of asymptomatic infections. Working as a cook, she was presumed to have infected 49 people, three of whom died.

Asymptomatic infections occur in both humans and animals. In fact, some of the notorious zoonoses arise from ostensibly asymptomatic animals (Ebolavirus-Bats [5], Ehrlichia-dogs [6], Brucella-sheep; Bartonella-cats). These animals may be completely asymptomatic, or just mildly ill, which may go unnoticed by the owner. While some microorganisms are transmitted to humans directly (airborne or food products), others are transmitted via an intermediary vector, such as ticks, or mosquitoes. Asymptomatic carriers of the pathogenic microbiota (animals or humans) constitute a reservoir maintaining their persistence in nature. The human gastrointestinal tract harbors prokaryotic cells at densities of 10 [11] cells $/ \mathrm{mL}$. These enteric cells have intimate functional and genetic relationship with both the local host and each other. These interactions probably keep the virulence of the organisms at bay, while maintaining the potential for infectivity (e.g. Poliovirus, Norovirus) [7]. Carriage of viruses is not rare; it has been estimated that each person may be concurrently infected with 10 different viruses, notably herpesviruses [8]. These viruses are transferable via airborne spread, body contact, blood transfusion, or vertically from mother to fetus.

How can asymptomatic infections be explained? Why do some people will not develop symptoms, yet clearly show serological conversion? Phylogenetically speaking, it can be argued that all creatures share the same archaic ancestor. As the phylogenetic tree has evolved over millions of years, some antigenic epitopes were lost, many were added, others have changed, but some remained conserved. Consequently, immunologically speaking, microorganisms belonging to one kingdom may share epitopes with microorganisms from another. This could partly explain why exposure to one microorganism may elicit an immune response which protects against a challenge with heterotypic microorganisms rendering the second infection asymptomatic [9]. Additional silencing mechanisms may be attributed to the less well-characterized cellular immune arm [10]. The recent developments in genetics have demonstrated the striking influence exerted by our genetic makeup on symptom development following an infectious challenge [11]. Speaking from a microbiological point of view, asymptomatic infections may be influenced either by a low inoculum size, or by low virulence microorganisms. Emerging data indicate that enteric viruses regulate, and are regulated by microbes in the gut through a process termed transkingdom interaction [12].

Why are asymptomatic infections important? Firstly, asymptomatic infections may provide immunity [13], Secondly, asymptomatic infections during pregnancy may produce devastating illness in the newborn (Zika virus, T. gondii, or cytomegalovirus) [14,15], or trigger changes leading to cancer $(H$. pylori, Hepatitis $\mathrm{B}$ virus, EBV, Papillomavirus). Thirdly, mice latently infected with gammaherpesvirus were resistant to infection with bacterial pathogens such as Listeria and Yersinia [7]. Fourth, with some microorganisms, failure to recognize their emergence in real-time may perpetuate epidemics, since a substantial proportion of transmissions occurs during the pre-symptomatic phase of infections (e.g. influenza, hepatitis A) [16]. Thus, timely diagnosis and perhaps treatment of asymptomatic infections is of prime importance.

This review will try to shed light on epidemiologic features of longknown and emerging pathogens known to cause AI, their importance in terms of public and personal health, and the immunologic mechanisms operating behind the scenes in a sample of parasites, bacteria, and viruses. Admitting these issues should foster efforts in diagnostics, public health, neonatology, vaccinology and perhaps therapeutics.

\section{Definitions}

An asymptomatic or subclinical infection may be defined, with a high degree of certainty, when an outbreak has been investigated in real time and certain exposed people, while not manifesting any symptom, demonstrate seroconversion [3]. However, when the timing "Corresponding Author: Prof. Israel Potasman, Maccabi $\mathrm{HMO}$, and the Rappaport Faculty of Medicine, 56a Hayam Rd., Haifa 3474407, Israel; E-mail: potasman@gmail.com

Citation: Potasman I (2017) Asymptomatic Infections: The Hidden Epidemic. Int J Clin Res Trials 2: 118. doi: https://doi.org/10.15344/2456-8007/2017/118

Copyright: () 2017 Potasman. This is an open-access article distributed under the terms of the Creative Commons Attribution License, which permits unrestricted use, distribution, and reproduction in any medium, provided the original author and source are credited. 
of infection is remote, the term asymptomatic may not always apply due to recall bias. For example, a pregnant woman with IgG antibodies against $T$. gondii may have been infected in childhood, making it difficult if not impossible to ascertain the asymptomatic nature of infection. Thus, acute or recent asymptomatic cases more clearly fit the definition of true asymptomatic infections.

A rather difficult hurdle in the terminology is how to define carriage of a potential pathogen such as $H$. pylori, Meningococcus or MRSA. Quite often, the terms asymptomatic infection and carriage overlap. At times, carriage of a pathogen may advance to full blown disease. The agents included in this review represent a convenience sample: they were chosen either because they have a significant impact and/or enough data has accumulated on these infections.

\section{Asymptomatic Protozoan Infections}

\section{Malaria}

The World Health Organization's (WHO) fact sheet on malaria asserts that almost half of the world's population in 2015 were at risk of contracting the disease. Owing to massive efforts, the global malaria incidence between 2000 and 2015 fell by $37 \%$ and the death rate by $60 \%$. According to the latest WHO estimates, there were 214 million cases of malaria in 2015 and 438,000 deaths [17]. Although older people in endemic areas may have asymptomatic parasitaemia, other patient-groups are prone to develop severe disease: infants, children under 5 years, pregnant women and patients with HIV/ AIDS. Malaria is probably the best studied parasitic disease depicting asymptomatic infection. Despite the absence of a standard definition of asymptomatic parasitaemia, most researchers regard this state as detection of asexual or sexual parasites in the blood with absence of symptoms [18]. The method employed to diagnose malaria (microscopy vs. antigenemia vs. PCR), and the varying sensitivities of each method will ultimately determine the rate of asymptomatic infection. Lindblade and colleagues [19] rightly regarded asymptomatic parasitaemia in malaria as the silent threat. They argue that since treatment is usually provided to care-seeking patients, while a large proportion of malaria infections are asymptomatic, the global malaria epidemic is self-perpetuating.

The rate of asymptomatic infections varies by malaria subtype. Using microscopy for diagnosis, a larger proportion of $P$. falciparum infections (37.5\%) compared with P. vivax infections (18.5\%) in Brazil were asymptomatic [20]. Age, blood parasite density, concomitant illness, and immunity are cardinal factors determining whether malarial infection will be asymptomatic or not. In a survey of children in Mozambique, children $<1$ year of age had the highest rate of fever attributed to malaria (15.1\%; as compared to $5.9 \%$ among 5-7 year olds). Children with a low-density P. falciparum parasitemia (<499 parasites $/ \mu \mathrm{L}$ ) had a lower rate of fever $(7.2 \%)$ as compared with children whose parasite densities were $\geq 50,000 / \mu \mathrm{L}$ (42.1\%) [21].

The importance of an intact immune system to combat malaria was demonstrated in a study of HIV infected patients. Those who had a lower count of CD4 had a higher likelihood of fever [22]. Others have found that IgG subclasses affected malaria symptoms, with children having elevated IgG1 antibodies showing better protection against severe malaria [23].

The role of the immune system in potentially keeping malaria under check for decades is best exemplified by the case of the 74-year-old Greek woman who had recrudescent P. malaria infection after decades of being symptomless [24]. Suffering from splenomegaly, she received a course of methotrexate (for suspected lymphoma). This was quickly followed by a quartan fever pattern. Her P. malaria infection was confirmed by antibody and RT-PCR assays.

\section{Toxoplasmosis}

T. gondii is a coccidian parasite of felids with humans and other warm-blooded animals as intermediate hosts [25] Historically, T. gondii infection has been considered asymptomatic in the vast majority of immunocompetent patients with only $10-20 \%$ of patients presenting with oligo-symptomatic cervical lymphadenopathy [25]. However, in the large outbreak associated with municipal drinking water in Victoria, BC, just 18\% of seropositive patients were symptomfree [26]. Furthermore, a systematic review of 38 selected outbreaks found a fairly constant figure of symptom-free patients ( 20\%) [27].

What are the presumed mechanisms which silence acute toxoplasmosis? One way to answer this query requires understanding of the serological diagnosis of toxoplasmosis. In most tests (either agglutination, or the Sabin-Feldman dye test, or ELISA) the serum is diluted before testing. In fact, dilution is necessary to minimize the effect of the so-called cross-reacting antibodies. Western-blot (WB) study of these cross-reacting antibodies demonstrated that each seronegative individual has, in fact, antibodies against certain Toxoplasma epitopes [28]. The WB bands were both of IgG and IgM types and seemed to appear in early childhood. It was therefore hypothesized that these naturally occurring antibodies could be part of the mechanisms responsible for silencing the clinical picture of acute toxoplasmosis.

In parallel to the humoral arm, the cellular immune system ( $\mathrm{T}$ cells, macrophages and type 1 cytokines) play a crucial role in the protection against toxoplasmosis and probably in transforming overt- into asymptomatic toxoplasmosis. Studies in mice revealed an expansion of both NK cells and $\Upsilon \delta$ T cells during early infection [29]. This expansion has been proposed to provide innate resistance. T-cells protect the host by secreting gamma INF, IL-2 and TNF- $\alpha$ [29]. Dendritic cells and macrophages also play a pivotal role in control of acute infection by early production of IL-12. A clinical correlate of the above can be found in ocular toxoplasmosis. Patients without ocular toxoplasmosis, as opposed to those with eye involvement had higher levels of IL-12 and INF- $\Upsilon$ [30].

\section{Asymptomatic bacterial infections}

\section{$Q$ fever}

Coxiella burnetii is an obligate intracellular organism that causes both acute and chronic Q fever (QF). QF is a zoonotic illness easily transmitted to man by aerosol, and thus is considered an agent of bioterrorism. As of this review, more than 550 articles alluding to outbreaks of QF have been reported in PubMed. Large outbreaks, which constitute a formidable opportunity to study asymptomatic infections have occurred in European countries: The Netherlands, Switzerland, UK, Germany, and France. In the largest outbreak in the Netherlands, $80-86 \%$ of those tested had clinical illness, with $15-20 \%$ defined as asymptomatic [2]. During the large outbreak in Germany (2003) $21 \%(6 / 29)$ of vulnerable pregnant or cardiac patients tested positive, but were asymptomatic [31]. A more recent study from the Netherlands have found that 23/122 patients with vascular QF (grafts, aneurysms) had asymptomatic infection [32]. These figures certainly raise the question, what are the determinants that drive $\mathrm{QF}$ to be an asymptomatic one? 
Age is probably one of these determinants. Children seem to be less symptomatic than adults [33]. During the outbreak in Switzerland in 1983 , children $<15$ years old accounted for $19 \%$ of the 415 seropositive cases, but only $5 \%$ of the 191 symptomatic cases. Overall, 70/80 children were asymptomatic, vs. 121/335 of adults [33].

Upon inhalation, C. burnetii targets alveolar macrophages wherein it replicates in a lysosome-like parasitiphorous vacuole. Macrophages are unable to kill the organism which thus multiplies. C. burnetii seems to secrete effector proteins that control macrophage function [34]. Surprisingly, avirulent strains trigger a robust, early proinflammatory response characterized by secretion of TNFa, IL- 6 and mature IL- $1 \beta$. TNF production is specifically enhanced in patients who develop QF endocarditis.

Genetic traits surely exert an effect on disease presentation as studied by cytokine polymorphism [35]. Interferon- $\Upsilon+874 \mathrm{~T} / \mathrm{A}$, and the IL-10 -592C/A polymorphisms significantly affected both disease severity, cytokine protein levels and the duration of illness in three infections: EBV, QF, and Ross River Virus. This stood in sharp contrast to those with INF- $\Upsilon+874$ AA genotype and IL-10 -592CC genotype who suffered a milder disease.

\section{Asymptomatic Viral diseases}

\section{Cytomegalovirus}

Cytomegalovirus (CMV) has a worldwide distribution. The seroprevalence rates of healthy adults in the US reach probably $70 \%$, and may reach $100 \%$ in Africa [36]. Among Israeli army recruits it was found to differ by parental origin but was $60-84 \%$ [37]. The clinical features of acute CMV in the immunocompetent host may include fever, night sweats, nausea, cough and fatigue. Yet, asymptomatic infections are probably more common and may bear devastating consequences. For example, a recent study of 238 pregnant women from France has found that $79 \%$ were asymptomatic [38]. This statement holds true both for adults and neonates who acquire the virus perinatally [1].

Cytomegalovirus infection in neonates may cause sensorineural hearing loss, cognitive defects, and motor defects. The overall infection rate of CMV in neonates in the USA is $0.6 \%$ [39] but among mothers who seroconvert during pregnancy it is much higher. In a study which included 123 participants and tested the efficacy of hyperimmune globulin the rate of asymptomatic infection was $41.4 \%$ [40] this underscores the importance of serological follow-up.

Asymptomatic CMV has rarely been studied at the time of acute infection. Yet, Zanghellini and colleagues have followed 45 adolescents for 7.5 months for the acquisition of CMV [41]. Six of these 45 (13.3\%) seroconverted and all were asymptomatic. CMV DNA was detected by PCR in the plasma and WBCs in all positive patients. The virus was also isolated from urine in $59.2 \%$ over 80 weeks' follow up, from saliva in 3 subjects, and vaginal swabs in $2 / 5$. These data prove that asymptomatic viral shedders constitute an important source of ongoing infection in the community.

Immunity against CMV is tri-factorial: humoral, innate and adaptive. Three lines of evidence support the role of humoral immunity. First, in animals, immunization against glycoprotein B induces neutralizing antibodies. Second, in pregnancy, the probability of transmission of infection to the fetus greatly increases if the antibody response is of low avidity and poor neutralizing activity [1]. Finally, in solid-organ transplant recipients, primary infection is often more frequent and severe in seronegative rather than seropositive. The share of cellular immunity in CMV infection is likewise important. Natural killer cells were shown to play a role in clearance of murine $\mathrm{CMV}$ infection. Ablation of NK cells renders mice susceptible to lethal CMV infection [42].

\section{Rhinoviruses}

The remarkable improvements achieved in diagnostic assays over the past decade have increased our capability of identifying viruses that cause respiratory infections. Rhinoviruses are the causative agents of most common colds and are partly responsible for exacerbations of chronic obstructive pulmonary disease. Infections may be symptomatic or asymptomatic in both immunocompetent and immunocompromised patients.

Children may experience an average of 8-12 colds per year. After inoculation onto the nasopharynx the virus may cause disease and be shed thereafter for up to 21 days. Transmission is most effective via the oral route during the first five days of infection.

Srinivasan et al. have detected human rhinovirus (HRV) in 5 of 33 asymptomatic children before undergoing allogeneic hematopoietic cell transplantation [43]. Four patients had continued to shed HRV for $\geq$ two weeks, and two of the four later developed upper respiratory infections. Symptomatic infections with HRV are more common among young children as opposed to older children and adults [44] Peltola and others have found that among shedders aged $<7$ years, $12 / 13$ were symptomatic, while only $2 / 14$ of those aged $>16$ years were symptomatic. They concluded that the mechanisms that determine the appearance of clinical symptoms are probably of both viral and host origin.

To further elucidate the mechanism, Cohen et al. [11] inoculated 152 healthy adults with rhinovirus 39 . Sixty-nine percent of the volunteers developed infection, while only $22 \%$ developed clinical illness, which means that $47 \%$ suffered an asymptomatic infection. Additional investigation revealed that shorter telomeres (in either PBMC or T-cell subsets) were associated with greater odds of infection, independent of pre-challenge virus-specific antibody or demographics. Furthermore, CD8CD28 - was the only cell population in which shorter telomeres were associated with greater risk of clinical illness. The association between CD8CD28 - telomere length and infection increased with age. Telomere shortening in leukocytes, as occurs with aging, has implications for immunocompetence. Shortening in the cytolytic CD8 T-cells is especially important for cancer and viral infections. The rapid loss of telomere length in cytolytic CD8 T-cells causes cell senescence marked by loss of expression of CD28, a co-stimulatory molecule important for antiviral function.

\section{Zika virus}

Zika virus (ZIKV) infection that swept through South America is the most recent example of an emerging infection capable of causing devastating consequences [14]. The prevailing consensus is that acute Zika infection may cause microcephaly and Guillain-Barré syndrome $[45,46]$ Thus, it came as no surprise when the WHO declared a state of public health emergency of international concern. The most common symptoms of Zika infection include headache, mild fever, rash, conjunctivitis and arthralgia. However, a non-negligible 
proportion of those affected produce no symptoms. In The YAP island outbreak the symptomatic: asymptomatic ratio was estimated at 1:4.4 [47], while a recent seroprevalence conducted in French Polynesia have found a ratio of 1:1 among adults and 2:1 among children [48].

Asymptomatic Zika infections may have grave outcomes: firstpregnant women may fail to seek obstetric attention in due time, second-the virus may be carried by asymptomatic patients across borders and continents, thus perpetuating epidemics, third-It may be transmitted sexually [49] by an apparent asymptomatic patient and four- be transmitted by blood donations [50].

Studies of the immune response, specifically in asymptomatic Zika patients are unavailable. However, a few studies in symptomatic patient and mice shed light on important elements of the immune system [51-53] Studies in mice showed that an inhibitory antibody, ZIKV-117 broadly neutralized infection of ZIKV strains corresponding to African and Asian-American lineages. Monoclonal antibody based on ZIKV-117 treatment markedly reduced tissue pathology, placental and fetal infection, and mortality. Another study found that the most potent neutralizing antibodies targeted $\mathrm{E}$ protein domain III or quaternary epitopes on infectious virus [52]. Studies in immunocompetent mice infected with ZIKV have demonstrated a relatively mild disease which elicited both $\mathrm{CD} 4+$ and CD8+ effector cytokines and cytolytic molecules [53] It is still unclear which of these mechanisms is responsible for driving ZIKV infection into an asymptomatic one.

\section{Summary and Future Directions}

Asymptomatic infections are common. Moreover, for some of the infections like ZIKV, asymptomatic presentations are probably more common than overt febrile presentations. These asymptomatic infections pose a serious threat to the public either by perpetuating epidemics, or by damaging fetuses.

The developments achieved over the recent few decades in molecular biology, metagenomics, host-microbe immunology and rapid diagnostics have unmasked some of the secrets of asymptomatic infections. Generally speaking, the interplay between the pathogen's virulence and host's immunology is what drives the clinical presentation. Vaccines constitute a formidable example how manipulation of the immune system turns an overt, and at times lethal infection into a subclinical one. However, a few cardinal queries remain: At the current time of individualized medicine, can we distinguish people with specific vulnerability to infections? Can we study AI with the aid of attenuated vaccines? Should some of the asymptomatic infections be treated at all? Can the knowledge gained from metagenomics be translated into vaccinology?

The study of asymptomatic infections, although problematic to investigate in real-time, is an exciting field that may teach us important lessons on how to combat infections more effectively. Future studies should provide additional data on the genetic and immunologic determinants that drive a robust, yet discreet response against an invading pathogen.

\section{Competing Interests}

The author declare that no competing interests exist.

\section{References}

1. Gandhi MK, Khanna R (2004) Human cytomegalovirus: clinical aspects, immune regulation, and emerging treatments. Lancet Infect Dis 4: 725-738.

2. Hackert VH, van der Hoek W, Dukers-Muijrers $N$, de Bruin A, Al Dahouk S, et al. (2012) Q fever: single-point source outbreak with high attack rates and massive numbers of undetected infections across an entire region. Clin Infect Dis 55: 1591-1599.

3. Ekman CC, Chiossi MF, Meireles LR, Andrade Júnior HF, Figueiredo WM, et al. (2012) Case-control study of an outbreak of acute toxoplasmosis in an industrial plant in the state São Paolo, Brazil. Rev Inst Med Trop Sao Paulo 54: $239-44$

4. Lundstrom T, Sobel J (2001) Nosocomial candiduria: a review. Clin Infect Dis 32: 1602-1607.

5. Leroy EM, Kumulungui B, Pourrut X, Rouquet $P$, Hassanin A, et al. (2005) Fruit bats as reservoirs of Ebola virus. Nature 438: 575-576

6. Davoust B, Bourry O, Gomez J, Lafay L, Casali F, et al. (2006) Surveys on seroprevalence of canine monocytic ehrlichiosis among dogs living in the Ivory Coast and Gabon and evaluation of a quick commercial test kit dotELISA. Ann N Y Acad Sci 1078: 464-469.

7. Gentile G, Micozzi A (2016) Speculations on the clinical significance of asymptomatic viral infections. Clin Microbiol Infect 22: 585-588.

8. Barton ES, White DW, Cathelyn JS, Brett-McLellan KA, Engle M, et al. (2007) Herpesvirus latency confers symbiotic protection from bacterial infection. Nature 447: 326-329.

9. Ramsey KH, Cotter TW, Salyer RD, Miranpuri GS, Yanez MA, et al. (1999) Prior genital tract infection with a murine or human biovar of Chlamydia trachomatis protects mice against heterotypic challenge infection. Infect Immun. 67: 3019-3025.

10. Thaiss CA, Levy M, Suez J, Elinav E (2014) The interplay between the innate immune system and the microbiota. Curr Opin Immunol 26: 41-48.

11. Cohen S, Janicki-Deverts D, Turner RB, Casselbrant ML, Li-Korotky HS, et al. (2013) Association between telomere length and experimentally induced upper respiratory viral infection in healthy adults. JAMA 309: 699-705.

12. Pfeiffer JK, Virgin HW (2016) Transkingdom control of viral infection and immunity in the mammalian intestine. Science.

13. Potasman I, Rzotkiewicz S, Pick N, Keysay A (2000) Outbreak of Q fever following a Safari trip. Clin Infect Dis 30: 214-215.

14. Pacheco O, Beltrán M, Nelson CA, Valencia D, Tolosa N, et al. (2016) Zika virus disease in Colombia- a preliminary report. N Engl J Med.

15. Bischoff AR, Friedrich L, Cattan JM, Uberti FA (2016) Incidence of symptomatic congenital toxoplasmosis during ten years in a Brazilian hospital. Pediatr Infect Dis J 35: 1313-1316.

16. Patrozou E, Mermel LA (2009) Does influenza transmission occur from asymptomatic infection or prior to symptom onset? Public Health Rep 124: 193-196.

17. http://www.who.int/mediacentre/factsheets/fs094/en/

18. Laishram DD, Sutton PL, Nanda N, Sharma VL, Sobti RC, et al. (2012) The complexities of malaria disease manifestations with a focus on asymptomatic malaria. Malar J 11: 29.

19. Lindblade KA, Steinhardt L, Samuels A, Kachur SP, Slutsker L, et al. (2013) The silent threat: asymptomatic parasitemia and malaria transmission. Exper Rev Anti Infect Ther 11: 623-639.

20. da Silva-Nunes M, Ferreira MU (2007) Clinical spectrum of uncomplicated malaria in semi-immune Amazonians: beyond the symptomatic vs asymptomatic dichotomy. Memorias Do Instituto Oswaldo Cruz 102: $341-$ 347.

21. Mabunda S, Aponte JJ, Tiago A, Alonso P (2009) A country-wide malaria survey in Mozambique. II. Malaria attributable proportion of fever and establishment of malaria case definition in children across different epidemiological settings. Malar J 8: 74.

22. French N, Nakiyingi J, Lugada E, Watera C, Whitworth JA, et al. (2001) Increasing rates of malarial fever with deteriorating immune status in HIV1-infected Ugandan adults. AIDS 15: 899-906.

23. Ndungu FM, Bull PC, Ross A, Lowe BS, Kabiru E, et al. (2002) Naturally acquired immunoglobulin (Ig)G subclass antibodies to crude asexual Plasmodium falciparum lysates: evidence for association with protection for IgG1 and disease for IgG2. Parasite Immunol 24: 77-82. 
24. Vinetz JM, Li J, McCutchan TF, Kaslow DC (1998) Plasmodium malariae infection in an asymptomatic 74-year-old Greek woman with splenomegaly. N Engl J Med 338:367-71.

25. Montoya JG, Boothroyd JC, Kovacs JA (2010) Toxoplasma gondii. In: Mandell GL, Bennett JE, Dolin R, eds. Principles and Practice of Infectious Diseases. 7th ed. Philadelphia, USA. Churchill Livingstone.

26. Bowie WR, King AS, Werker DH, Isaac-Renton JL, Bell A, et al. (1997) outbreak of toxoplasmosis associated with municipal drinking water. The BC toxoplasma investigation team. Lancet 350: 173-177.

27. Meireles LR, Ekman CCJ, de Andrade HF, Luna EJA (2015) Human toxoplasmosis outbreaks and the agent infecting form. Findings from a systematic review. Rev Inst Med Trop Sao Paolo 57: 369-376.

28. Potasman I, Araujo FG, Remington JS (1986) Toxoplasma antigens recognized by naturally occurring human antibodies. J Clin Microbiol 24 1050-1054.

29. Hunter CA, Subauste CS, Van Cleave VH, Remington JS (1994) Production of gamma interferon by natural killer cells from Toxoplasma gondii-infected SCID mice: regulation by interleukin-10, interleukin-12, and tumor necrosis factor alpha. Infect Immun 62: 2818-2824.

30. Vallochi AL, Goldberg AC, Falcai A, Ramasawmy R, Kalil J, et al. (2008) Molecular markers of susceptibility to ocular toxoplasmosis, host and guest behaving badly. Clin Ophthalmol 2: 837-848.

31. Wagner-Wiening C, Brockmann S, Kimmig P (2006) Serological diagnosis and follow-up of asymptomatic and acute $\mathrm{Q}$ fever infections. Int J Med Microbiol 40: 294-296.

32. Broos PP, Hagenaars JC, Kampschreur LM, Wever PC, Bleeker-Rovers CP, et al. (2015) vascular complications and surgical interventions after world's largest Q fever outbreak. J Vasc Surg 62: 1273-1280.

33. Maltezou HC, Raoult D (2002) Q fever in children. Lancet Infect Dis 2: 686691.

34. Graham JG, MacDonald L, Hussain SK, Sharma UM, Kurten RC, et al. (2013) Virulent Coxiella burnetii pathotypes productively infect primary human alveolar macrophages. Cell Microbiol 15: 1012-1025.

35. Vollmer-Conna U, Piraino BF, Cameron B, Davenport T, Hickie I, et al. (2008) Cytokine polymorphisms have a synergistic effect on severity of the acute sickness response to infection. Clin Infect Dis 47: 1418-1425.

36. Crumpacker CS II, Zhang JL (2010) Cytomegalovirus. In: Mandell GL, Bennett JE, Dolin R, eds. Principles and Practice of Infectious Diseases. 7th ed. Philadelphia, USA. Churchill Livingstone

37. Green MS, Cohen D, Slepon R, Robin G, Wiener M, et al. (1993) Ethnic and gender differences in the prevalence of anti-cytomegalovirus antibodies among young adults in Israel. Int J Epidemiol 22: 720-723.

38. Picone O, Vauloup-Fellous C, Cordier AG, Guitton S, Senat MV, et al. (2013) A series of 238 cytomegalovirus primary infections during pregnancy: description and outcome. Prenat Diagn 33: 751-758.

39. Cannon MJ, Davis KF (2005) Washing our hands of the congenital cytomegalovirus disease epidemic. BMC Public health 5: 70.

40. Revello MG, Lazzarotto T, Guerra B, Spinillo A, Ferrazzi E, et al. (2014) A Randomized Trial of Hyperimmune Globulin to Prevent Congenital Cytomegalovirus. N Engl J Med 370: 1316-1326.

41. Zanghellini F, Boppana SB, Emery VC, Griffiths PD, Pass RF, et al. (1999) Asymptomatic primary cytomegalovirus infection: virologic and immunologic features. J Infect Dis 180: 702-707.

42. Polić B, Hengel H, Krmpotić A, Trgovcich J, Pavić I, et al. (1998) Hierarchical and redundant lymphocyte subset control precludes cytomegalovirus replication during latent infection. J Exp Med 188: 1047-1054

43. Srinivasan A, Flynn P, Gu Z, Hartford C, Lovins R, et al. (2013) Detection of respiratory viruses in asymptomatic children undergoing allogeneic hematopoietic cell transplantation. Pediatr Blood Cancer 60: 149-151.

44. Peltola V, Waris M, Osterback R, Susi P, Ruuskanen O, et al. (2008) Rhinovirus transmission within families with children: incidence of symptomatic and asymptomatic infections. J Infect Dis 197: 382-389.

45. http://www.who.int/mediacentre/factsheets/zika/en/

46. Martines RB, Bhatnagar J, de Oliveira Ramos AM, Davi HP, Iglezias SD, et al. (2016) Pathology of congenital Zika syndrome in Brazil: a case series. Lancet 388: 898-904

Int J Clin Res Trials

ISSN: 2456-8007
47. Duffy MR, Chen TH, Hancock WT, Powers AM, Kool JL, et al. (2009) Zika Virus Outbreak on Yap Island, Federated States of Micronesia. N Engl J Med 360: 2536-2543.

48. Aubry M, Teissier A, Huart M, Merceron S, Vanhomwegen J, et al. (2017) Zika Virus seroprevalence, French Polynesia, 2014-2015. Emerg Infect Dis.

49. Moreira J, Peixoto TM, Siqueira AM, Lamas CC (2017) Sexually acquired Zika virus: a systematic review. Clin Microbiol Infect 23: 296-305.

50. Galel SA, Williamson PC, Busch MP, Stanek D, Bakkour S, et al. (2017) First Zika-positive donations in the continental United States. Transfusion 57: 762-769.

51. Sapparapu G, Fernandez E, Kose N, Bin Cao, Fox JM, et al. (2016) Neutralizing human antibodies prevent Zika virus replication and fetal disease in mice. Nature 540: 443-447.

52. Stettler K, Beltramello M, Espinosa DA, Graham V, Cassotta A, et al. (2016) Specificity, cross-reactivity and function of antibodies elicited by Zika virus infection. Science 353: 823-826.

53. Pardy RD, Rajah MM, Condotta SA, Taylor NG, Sagan SM, et al. (2017) Analysis of the T-cell response to Zika virus and identification of a novel CD8+ T-cell epitope in immunocompetent mice. PLoS Pathogen 13: e1006184 